\title{
[CONCERNING THE ORDER OF THE FEMALE PERSONS]
}

concerning the order of the female persons the winning thought probably sprouted out of consideration towards the living spouse so the first place should have been the one closest in time therefore the third one so that the second and the first should also have the place according to time and that every daughter should stand directly behind her mother except the name of the first spouse barbara also the names of the second and of the third the second spouse's name was anastasia the third one was called agnes the daughter with anastasia gertrude and the woman from the marriage with barbara magdalena 